\title{
Efecto de la estrategia en el desempeño de la empresa. Un estudio en la industria manufacturera de Colombia
}

\author{
Serna Gómez, Héctor Mauricio* \\ Calderón Hernández, Gregorio** \\ Naranjo Valencia, Julia Clemencia***
}

\section{Resumen}

La orientación estratégica que adopta la empresa genera un efecto en el desempeño de la misma, por tal razón, el presente trabajo se traza como objetivo identificar el efecto que genera la orientación estratégica de la empresa sobre el desempeño de la misma. El estudio se realizó en 199 empresas industriales de Colombia, el procesamiento de la información se desarrolló bajo un análisis factorial confirmatorio -CFA- y un modelo de ecuaciones estructurales -SEM-. Se logra identificar tres tipos de configuraciones estratégicas, asimismo se identifica un efecto positivo de éstas sobre los indicadores financieros y sobre la percepción del desempeño. Se concluye que aspectos como racionalización y reducción de costos han pasado a un segundo plano en el contexto colombiano, mientras que se identifica una gran incidencia del desarrollo de procesos de marketing y calidad del producto en la configuración de la estrategia.

Palabras clave: Estrategia, recursos competitivos, desempeño organizacional, indicadores financieros, industria manufacturera colombiana.

Recibido: 20-03-11. Aceptado: 07-11-12

* $\quad$ Magíster en Economía, Universidad de Manizales; Administrador de empresas Universidad Nacional de Colombia, Manizales. Profesor Catedrático Facultad de Ciencias Contables, Económicas y Administrativas Universidad de Manizales. Integrante grupo Cultura Organizacional y Gestión Humana, categoría A en Colciencias, e-mail: hmsernag@unal.edu.co

** Doctor en Administración y Dirección de Empresas, Universidad Pablo de Olavide Sevilla-España; magister en desarrollo educativo y social, Universidad Pedagógica Nacional, Bogotá; magister en administración, Universidad EAFIT, Medellín; administrador de empresas Universidad Nacional de Colombia, Manizales. Profesor Tiempo Completo Facultad de Ciencias Contables, Económicas y Administrativas Universidad de Manizales. Integrante del grupo de investigación Cultura Organizacional y Gestión Humana, categoría A en Colciencias, e-mail: gcalderonh@unal.edu.co

*** Doctora en Ciencias de la Empresa, Universidad de Murcia, Murcia-España; Especialista en evaluación socioeconómica de proyectos de inversión, Universidad Nacional de Colombia, Manizales; Ingeniera Industrial, Universidad Nacional de Colombia, Manizales. Profesora asociada Universidad Nacional de Colombia, Manizales. Coordinadora del grupo de investigación Cultura Organizacional y Gestión Humana, categoría A en Colciencias, e-mail: jcnaranjov@unal.edu.co 


\title{
The Effect of Strategy on Company Performance. A Study of the Manufacturing Industry in Colombia
}

\begin{abstract}
The strategic orientation adopted by a company generates an effect on its performance; the objective of this work is to identify the effect that a company's strategic orientation has on its performance. The study was carried out at 199 industrial companies in Colombia; information was processed using confirmatory factor analysis, CFA, and a structural equations model, SEM. Three types of strategic configurations were identified as were the positive effect these had on financial indicators and the perception of performance. Conclusions were that aspects such as cost rationalization and reduction have faded to the background in the Colombian context, while a high incidence for the development of marketing processes and product quality was identified in configuring the strategy.
\end{abstract}

Key words: Strategy, competitive resources, organizational performance, financial indicators, Colombian manufacturing industry.

\section{Introducción}

La estrategia se considera clave para el logro de ventajas competitivas sostenibles por cuanto articula la forma en que la organización encara sus fortalezas y debilidades y configura sus actividades de mercado para ganar dicha ventaja (Sheppeck y Militello, 2000). La literatura empírica señala la relación entre la estrategia adoptada por la empresa y sus efectos en el desempeño medido a nivel tanto cuantitativo (Dess y Davis, 1984; Robinson y Pearce, 1988) como cualitativo (Aragón, 2004; Camisón y Boronat, 2004; Calderón et al., 2009a, 2009b, 2011a y 2011b).

No obstante, en el ámbito colombiano no se tiene referencia de trabajos que evalúen esta relación, con excepción de los trabajos de Calderón et al. (2009a, $2009 b, 2011$ a y 2011b) en los cuales, se identifican relaciones entre el área de gestión humana con la estrategia adopta- da por la empresa (Calderón et al., 2009a) y se establecen orientaciones estratégicas de acuerdo al peso dado a sus factores competitivos (Calderón et al., 2009b).

La presente investigación pretende dar un paso adelante evaluando el efecto causal de las configuraciones estratégicas en los indicadores de desempeño organizacional. Es de resaltar como aporte de esta investigación, que la relación causal aquí planteada no ha sido testeada en otros contextos, dado que otras investigaciones presentan relaciones a doble vía entre las variables y no identifican la incidencia que genera la estrategia sobre el desempeño (Porter, 1980; Dess y Davis, 1984; Robinson y Pearce, 1988), asimismo se resalta el cambio de enfoque en el uso del análisis factorial realizado en la presente investigación en comparación a investigaciones anteriores de los mismos autores (Calderón et al., 2009a, 2009b, 
2011a y 2001b) el cual permite identificar hallazgos antes no encontrados.

Teniendo en cuenta que el estudio pretende encontrar el efecto de la estrategia en el desempeño de la empresa manufacturera colombiana, se enviaron cuestionarios a toda la población objeto de estudio ${ }^{1}$, se logró obtener respuesta de 199 empresas. Se verificó la consistencia de la muestra a través de un análisis de varianza ANOVA que evaluó aspectos como: ventas, activos, patrimonio y la relación ebitda/ventas, esto permitió comprobar que no existen diferencias significativas entre las empresas que respondieron los cuestionarios y las que no lo hicieron.

Para la recolección de información se utilizaron dos cuestionarios, el primero pretendía recopilar información de los factores competitivos a través de la medición de 22 ítems adaptados de los trabajos de Dess y Davis (1984), Robinson y Pearce (1988) y Camelo et al. (2000, 2004). El segundo cuestionario pretendía recopilar información perceptual sobre el desempeño organizacional a través de
12 ítems adaptados de la investigación de Gupta y Govindarajan (1984).

La escala de medición de los dos cuestionarios fue una escala tipo likert de uno a cinco, donde uno representaba la peor valoración y cinco representaba la mejor valoración. Es importante resaltar que se identificó la validez y confiabilidad de los instrumentos utilizados, aunque estos ya habian sido validados para el contexto colombiano (Calderón et al., 2009a y 2009b), inicialmente se realizó una validez de contenido por parte de expertos, luego se identificó la consistencia interna identificando el alpha de cronbach $^{2}$, por medio del cual se establece que las dimensiones propuestas para analizar la estrategia empresarial y el desempeño organizacional presentan medidas homogéneas, también se identificó la validez convergente ${ }^{3} y$ la validez discriminante ${ }^{4}$.

El presente estudio plantea tres hipótesis que suponen el desarrollo de tipologías estratégicas en la industria manufacturera colombiana y la existencia de un impacto positivo de ésta en los indicadores financieros como ingresos por ven-

$1 \quad$ La población objeto de estudio son empresas manufactureras con un rango entre 200 y 1000 empleados, los cuales fueron identificadas a través de la base de datos 5000 empresas más grandes de Colombia.

2 El alpha cronbach se utilizó para evaluar los instrumentos y las dimensiones propuestas para medir la estrategia empresarial y el desempeño organizacional es acorde con el valor teórico $(\alpha>0,7)$.

3 Se identificaron las correlaciones entre las dimensiones para cada cuestionario (estrategia empresarial y desempeño organizacional) obteniendo como resultado correlaciones significativas $(\rho<0,05)$ en ambos casos, lo que corrobora la convergencia de las dimensiones para evaluar un mismo constructo.

4 Se compararon las correlaciones entre los ítems de cada dimensión con las correlaciones entre los ítems de otras dimensiones para cada instrumento, lo cual identifica la estructuración de los cuestionarios en dimensiones. 
Efecto de la estrategia en el desempeño de la empresa...

Serna G., Héctor; Calderón H., Gregorio y Naranjo V., Julia

tas, ingresos antes de intereses, impuestos, depreciaciones y amortizaciones -EBITDA-, retornos financieros -ROE-, retorno sobre activos ROA y en la percepción del desempeño. La contrastación de las hipótesis se desarrolló mediante un análisis factorial confirmatorio -CFA- y un modelo de ecuaciones estructurales -SEM-.

\section{La estrategia empresarial}

El concepto de estrategia tiene sus inicios en los trabajos de Barnard (1938), Selznick (1957), Penrose (1959) y Andrews (1971) quienes coinciden en que el éxito de las organizaciones se debe al buen manejo y uso de los recursos internos de la organización, y específicamente a la organización interna de los procesos, pero es con Chandler (1962) que el concepto de estrategia toma fuerza, identificándola como:

"La base de la determinación de las metas y los objetivos de una empresa a largo plazo, así como la adopción de cursos de acción y la asignación de los recursos necesarios para llevara a cabo los objetivos" (Chandler, 1962:13).

Asimismo trabajos como los de Ansoff (1965) y Andrews (1971) afirman el concepto esclarecido por Chandler (1962). El primero define la estrategia como la base para la consecución de las metas y los objetivos, el segundo plantea la noción de estrategia como cooperación e interdependencia entre las unidades de negocio, y Andrews (1971) la describe como el patrón de políticas, planes y metas para la consecución de los objetivos de forma tal que es posible identificar en qué lugar se encuentra la empresa y qué tipo de empresa es o va a ser. Los trabajos antes mencionados dan prevalencia a una perspectiva contingencial, en la cual, la estrategia depende del efecto combinado de una serie de características internas y externas a la organización (Venkatraman y Camillus, 1984).

Desde la perspectiva de la organización industrial se plantea que la estructura de la industria determina la naturaleza de su competencia en una industria, y la naturaleza de la competencia determina los resultados de la empresa (Camisón y Boronat, 2004) lo cual lleva a plantear el concepto de grupos estratégicos (Hunt, 1972; Newman, 1978) definido éste como un grupo de empresas del mismo sector con las mismas o similares estrategias (Porter, 1980) y se establece que la rentabilidad de la empresa está en función de la estructura de la industria y de su conducta estratégica (Hatten y Schendel 1977; Hatten et al., 1978; Cool y Schendel, 1987).

Derivado de lo anterior, la literatura ha desarrollado tipologías para determinar el curso de acción de la empresa, a partir de la estructura y los procesos de la organización -perspectiva contingencial- (Ansoff, 1965; Ansoff y Brandenburg, 1971; Segal, 1974; Anderson y Paine, 1975; Miles et al., 1978) y otros las han desarrollado a partir de la estructura y el desempeño de la industria -perspectiva de la organización industrial- (Caves y Porter, 1977; Porter, 1980, 1996).

Segal (1974) desarrolla una tipología estratégica desde la estructura organizacional identificando tres tipos de organizaciones: organización estructurada en cadena, organización medianamente estructurada, y organización adaptativa- 
mente estructurada. La primera se caracteriza por presentar una estrategia inherente a la tarea y por percibir el ambiente como estático y homogéneo, la segunda establece una estrategia para desarrollar cambios incrementales en las tareas y percibe el ambiente como una composición de entidades y procesos, y la última genera estrategias con grandes variaciones y con alta adopción del riesgo y percibe el ambiente de manera turbulenta y compleja.

Miles et al. (1978) identifican tres tipologías estratégicas: defensivas, prospectivas y analizadoras. La primera pretende mantener un ambiente estable para la organización para minimizar riesgos, la segunda busca establecer un ambiente innovador y dinámico para la organización para generar mayor rentabilidad, y la tercera pretende minimizar los riesgos del ambiente mientras genera oportunidades para maximizar su rentabilidad.

Caves y Porter (1977) y Porter $(1980,1996)$ desarrollan un modelo estructural centrado en la competencia intra-industria, en el cual se reconoce el papel de la industria para la formulación de una estrategia, asimismo identifica estrategias genéricas -liderazgo en costo, diferenciación y segmentación- las cuales, pueden ser usadas para ubicar la empresa en una industria en particular $y$, por consiguiente, construir una ventaja competitiva. Sin embargo, en esta línea de investigación de la estrategia se perciben como debilidad desde los aspectos teóricos una ausencia de análisis de factores potencialmente predominantes del desempeño como la estructura, complejidad y dinamismo de la organización (Bar- ney y Hoskisson, 1990; Camisón y Boronat, 2004).

Posteriormente con los trabajos fundamentados en la teoría de recursos y capacidades (Wernerfelt, 1984; Grant, 1991; Barney, 1991) se fortalece un aspecto que ha sido fundamental en el campo de la estrategia: su importancia para construir ventaja competitiva sostenida y en consecuencia para obtener un rendimiento superior al promedio (Hitt et al., 2008). Lo anterior ha permitido establecer entre diversos académicos un acercamiento desde la perspectiva contingencial al modelo de grupos estratégicos formulado por Porter (Dess y Davis, 1984).

Entre los autores que asumieron esta última, retomando aspectos contingenciales, están Camisón y Boronat (2004) quienes plantean que tanto el entorno competitivo en que la empresa se localiza, como las competencias distintivas de la organización afectan positivamente el desempeño; y Dess y Davis (1984), que plantean que las empresas se diferencian por la manera en que utilizan los factores competitivos para asumir decisiones estratégicas y como consecuencia de ello se conforman conjuntos empresariales, algunos de los cuales pueden presentar mejores resultados que otros.

Es de resaltar que los factores desarrollados por Dess y Davis (1984) han sido validados en otros contextos (Venkatraman y Grant, 1986; Ketchen et al., 1997; Camisón et al., 2007; García-Borbolla et al., 2009), y han sido validados en el contexto colombiano (Calderón et al., 2009a y 2009b) por tanto, se consideran pertinentes para la presente investigación. 
Efecto de la estrategia en el desempeño de la empresa...

Serna G., Héctor; Calderón H., Gregorio y Naranjo V., Julia

Cabe acotar que en los trabajos previos desarrollados en el contexto colombiano se analizaron los factores competitivos para establecer aspectos relacionados con la estrategia desde una perspectiva de grupos estratégicos (Calderón et al., 2009a, 2009b, 2011a y $2011 b)$, mientras que la presente investigación analiza la configuración de factores competitivos en la organización, es decir, en cada una de las empresas, lo cual permite enunciar la siguiente hipótesis:

Hipótesis 1: el peso otorgado a los factores competitivos genera estrategias genéricas en las empresas colombianas.

\section{Relación estrategia y desempeño}

La literatura empírica se ha interesado por estudiar la importancia de la estrategia en el desempeño de las empresas (Benett et al., 1998; Bird y Beecheler, 1995; Porter, 1980; Akan et al., 2006). Hatten et al. (1978) identifican la relación entre el desempeño financiero y la estrategia adoptada, Schendel y Hofer (1979) plantean que las mejores estrategias generan impacto en el desempeño, Woo y Cooper (1981) señalan que la implementación de la estrategia difiere significativamente del mercado en que la empresa participa y asimismo difiere su desempeño financiero, MacMillan et al. (1982) establecen que aspectos estratégicos comunes de las unidades de negocio de la empresa tienen una relación significativa con la rentabilidad, por su parte Porter (1980) evaluó la incidencia de la estrategia en el desempeño organizacional, bajo la premisa de estrategias genéricas.

Por otra parte, la teoría de recursos y capacidades identifica que las empresas con desempeño superior desarrollan una ventaja competitiva cuando favorecen sus recursos internos (Wernerfelt, 1984; Hansen y Wernerfelt, 1989; Rumelt, 1991), es decir que la rentabilidad se encuentra en la propia empresa y no en el sector industrial donde esta se ubica (Barney, 1986, 1997; Peteraf, 1993; Grant, 1991). De acuerdo con lo anterior, autores como Dess y Davis (1984) y Robinson y Pearce (1988) reconocen que las ventajas competitivas se desarrollan desde las estrategias genéricas, las cuales son formuladas desde el direccionamiento estratégico (Hambrick y Mason, 1984; Hill y Jones, 2005).

Con respecto a las medidas utilizadas para medir el desempeño organizacional, en la literatura se identifican diferentes medidas financieras como retorno sobre inversión ROI (Sánchez y Aragón, 2003), retorno sobre activos ROA (Carpenter y Sanders, 2002), rentabilidad (Desphandé et al., 1993; Hill y Jones, 2005) y beneficios (Aragón et al., 2003).

Así mismo se han propuesto medidas cualitativas que buscan dar cuenta de la evolución de diversas variables empresariales (Aragón, 2004). Específicamente para analizar la efectividad de las organizaciones se ha considerado la medida de desempeño, propuesta por Quinn (1988) como un constructo asociado con diferentes modelos de análisis organizacional -modelo de proceso interno, modelo del sistema abierto, modelo racional, y modelo de relaciones humanas- adapta- 
da del modelo de valores en competencia de Quinn y Rohrbaugh $(1981,1983)$. El modelo analiza coordinación de los procesos internos, la organización de las tareas del personal, la calidad del producto, la satisfacción de los clientes, la habilidad de adaptación a las necesidades cambiantes del mercado, la imagen de la empresa y sus productos, la cuota de mercado, la rentabilidad, la productividad, la motivación de los trabajadores y el absentismo.

Por su parte, Gupta y Govindarajan (1984) proponen 13 dimensiones de desempeño: ventas, tasa de crecimiento, proporción de mercado, rentabilidad operativa, rentabilidad en ventas, flujo de caja de las operaciones, retorno sobre la inversión, desarrollo de nuevos productos, desarrollo de mercados, actividades de I+D, programas de reducción de costos, desarrollo de personal y asuntos políticos y públicos.

Para estudiar el efecto de la estrategia en el desempeño organizacional en el presente estudio se opta por analizar indicadores financieros -ROA, ROE, EBITDA- y la medida de percepción de efectividad, la cual ha sido validada para el contexto colombiano (Calderón et al., 2009a y 2009b). En general, los hallazgos de estos estudios previos (Dess y Davis, 1984; Hambrick y Mason, 1984; Wernerfelt, 1984; Robinson y Pearce, 1988; Hansen y Wernerfelt, 1989; Rumelt, 1991; Camisón y Boronat, 2004; García et al., 2009) permiten predecir que el desarrollo de estrategias a partir de la configuración de sus factores competitivos generan un impacto positivo sobre el desempeño de la empresa, tanto a nivel cuantitativo y cualitativo, lo cual permite enunciar las siguientes hipótesis:

Hipótesis 2: la estrategia desarrollada por la empresa a partir del peso otorgado a sus métodos competitivos generan un impacto positivo en los indicadores financieros de la empresa - ventas, EBIT-DA, ROE, ROA-.

Hipótesis 3: la estrategia desarrollada por la empresa a partir del peso otorgado a sus métodos competitivos generan un impacto positivo en la percepción del desempeño organizacional.

\section{Efecto de la estrategia en el desempeño de la empresa}

Para reconocer el efecto de estrategia en el desempeño de la empresa, es importante identificar los ítems de medición de los factores competitivos (Cuadro 1) y de la medida perceptual de desempeño (Cuadro 2). Los ítems para medir los factores competitivos fueron adaptados de los trabajos de Dess y Davis (1984), Robinson y Pearce (1988) y Camelo, et al. (2000, 2004), los cuales, han sido validados en otros contextos (Venkatraman y Grant, 1986; Ketchen et al., 1997) y en el contexto colombiano (Calderón et al., 2009a y 2009b). En el Cuadro 1 se detallan los ítems de medición de los factores competitivos.

Para medir el desempeño organizacional se asumen los indicadores financieros -ROE, ROA, EBITDA- de las empresas estudiadas, y el índice de percepción de efectividad de Gupta y Govindarajan (1984) el cual ha sido validado para el contexto colombiano (Calderón et 
Efecto de la estrategia en el desempeño de la empresa...

Serna G., Héctor; Calderón H., Gregorio y Naranjo V., Julia

\section{Factores competitivos}

\begin{tabular}{cl}
\hline Código & \\
\hline FC1 & Calidad del producto \\
FC2 & Desarrollo de nuevos productos \\
FC3 & Productividad del Negocio \\
FC4 & Esfuerzo continuo de reducción del costo \\
FC5 & Esfuerzo riguroso en el establecimiento de procedimientos de control de calidad del producto \\
FC6 & Precio \\
FC7 & Gama amplia de productos \\
FC8 & Esfuerzo por lograr una marca identificable \\
FC9 & Influir en los canales de distribución \\
FC10 & Esfuerzos por mejorar la validez de las materias primas \\
FC11 & Innovación en el proceso de fabricación \\
FC12 & Capacidades amplias de servicio al cliente \\
FC13 & Iniciativas concretas para lograr un equipo humano adiestrado y experimentado \\
FC14 & Mantenimiento de niveles bajos de inventario \\
FC15 & Mejora de los productos existentes \\
FC16 & Innovación en técnicas y métodos de marketing \\
FC17 & Promoción y Publicidad por encima de la media del sector \\
FC18 & Capacidad para fabricar productos especializados \\
FC19 & Especialización en segmentos geográficos \\
FC20 & Productos en segmentos de alto precio \\
FC21 & Esfuerzos para mejorar la calidad de la publicidad \\
FC22 & Esfuerzos para alcanzar una reputación \\
\hline
\end{tabular}

Fuente: Calderón et al. (2009a y 2009b).

al., 2009a y 2009b). En el Cuadro 2 se detallan los ítems de medición de la percepción de efectividad organizacional.

Para la comprobación de las hipótesis propuestas se realizó un análisis factorial confirmatorio bajo el método de rotación oblicuo ${ }^{5}$, el cual identifica la exis- tencia de cinco factores significativos (autovalores $\geq 1$ ) pero sólo tres factores explican un alto porcentaje de la varianza total explicada. Los otros dos factores hallados fueron excluidos por el interés de parsimonia del modelo, también se excluyen 3 de los 22 ítems porque no represen-

5 Se utilizó el método oblicuo por las características de los datos y porque no se realizó tipificación de estos; asimismo porque genera resultados con posibilidad de no correlación, lo cual permite que el modelo SEM sea evaluado bajo aspectos de no colinealidad. 


\begin{tabular}{ll} 
& \multicolumn{1}{c}{$\begin{array}{c}\text { Cuadro } 2 \\
\text { Ítems de medición de la } \\
\text { percepción de efectividad } \\
\text { organizacional }\end{array}$} \\
\hline \multicolumn{1}{c}{ Código } & \multicolumn{1}{c}{ Categorías } \\
\hline EO1 & Ventas \\
EO2 & Tasa de crecimiento \\
EO3 & Cuota de mercado \\
EO4 & Ganancias operativas \\
EO5 & Ganancias por ratio de ventas \\
EO6 & Flujo de caja de operaciones \\
EO7 & Retorno sobre la inversión \\
EO8 & Desarrollo de nuevos productos \\
EO9 & Desarrollo de mercados \\
EO10 & Actividades de l+D \\
EO11 & Programas de reducción de costos \\
EO12 & Desarrollo de personal \\
\hline
\end{tabular}

Fuente: Calderón et al. (2009a y 2009b).

tan una varianza significativa en los factores hallados $(\rho \leq 0.40)$ (Nunnally, 1978). Por último se realiza la rotación de los factores por medio del método Oblimin (ver Tabla 1). Lo que genera en la presente investigación la conformación de 3 configuraciones estratégicas, las cuales están construidas por 19 de los 22 factores competitivos.

Para comprobar la bondad y ajuste del modelo propuesto, se realizó el análisis de correlación de los residuos de los ítems y un análisis de la matriz de correlación anti-imagen, que permiten establecer que la muestra es adecuada, y por tanto las cargas factoriales son estables. Es de precisar que 3 ítems del cuestionario no presentan cargas factoriales superiores a 0.4 en ninguno de los factores identificados, por tanto, se descartan del análisis.

La primera configuración está compuesto por 6 de las 19 variables, que se caracterizan por el esfuerzo continuo por la reducción de costos, y por la focalización del mercado (desarrollo de amplia gama de productos, el esfuerzo por lograr una marca identificable, el esfuerzo por influir en canales de distribución, el desarrollo de técnicas y métodos de marketing, la promoción y publicidad, y la especialización en segmentos geográficos), por tanto, se denomina focalización del mercado.

La segunda configuración está compuesta por 8 de las 19 variables, y se caracterizan por la calidad del producto, la productividad del negocio, esfuerzo continuo de la reducción del costo, el esfuerzo riguroso en el establecimiento de procedimientos de control de calidad del producto, el esfuerzo por mejorar la validez de las materias primas, la mejora de productos existentes, la conformación de un precio competitivo, y la existencia de capacidades de servicio al cliente, por lo que se denomina calidad del producto.

El tercer y último factor está compuesto por 5 de las 19 variables, que se caracteriza por la innovación en el proceso de fabricación, el desarrollo de iniciativas concretas para lograr un equipo humano adiestrado y experimentado, la capacidad para fabricar productos especializados, la conformación de productos en segmentos de alto precio, y el esfuerzo para alcanzar una reputación, por tanto se denomina innovación en producto (ver Cuadro 3).

Esto lleva a identificar configuraciones de factores estratégicos de acuerdo al peso dado a los factores competitivos por las empresas colombianas, lo cual confirma la hipótesis 1.

Es de resaltar que los resultados sobre las configuraciones estratégicas 
Efecto de la estrategia en el desempeño de la empresa...

Serna G., Héctor; Calderón H., Gregorio y Naranjo V., Julia

Tabla 1

Matriz de componentes principales rotados de los factores competitivos

\begin{tabular}{ccccc}
\hline Variable & Factor $\mathbf{1}$ & Factor $\mathbf{2}$ & Factor $\mathbf{3}$ & Comunalidades \\
\hline FC01 & 0.0055 & $\mathbf{0 . 6 9 6}$ & 0.143 & 0.4950 \\
FC02 & 0.3224 & 0.3324 & 0.2821 & 0.7060 \\
FC03 & 0.0089 & $\mathbf{0 . 7 6 8 1}$ & 0.0485 & 0.4075 \\
FC04 & 0.0815 & $\mathbf{0 . 7 4 0 3}$ & -0.0193 & 0.4449 \\
FC05 & 0.2436 & $\mathbf{0 . 7 2 1 3}$ & 0.267 & 0.3491 \\
FC06 & 0.1754 & $\mathbf{0 . 4 5 1 1}$ & 0.0226 & 0.7652 \\
FC07 & $\mathbf{0 . 6 0 3 3}$ & 0.176 & -0.0693 & 0.6003 \\
FC08 & $\mathbf{0 . 6 6 1 5}$ & 0.3643 & 0.1754 & 0.3989 \\
FC09 & $\mathbf{0 . 7 5 8}$ & 0.2337 & 0.0745 & 0.3652 \\
FC10 & 0.2479 & $\mathbf{0 . 5 1 3 7}$ & 0.385 & 0.5264 \\
FC11 & 0.2175 & 0.3942 & $\mathbf{0 . 5 2 3 1}$ & 0.5236 \\
FC12 & 0.2448 & $\mathbf{0 . 5 5 2 5}$ & 0.5312 & 0.3527 \\
FC13 & 0.2116 & 0.3699 & $\mathbf{0 . 5 8 7 4}$ & 0.4734 \\
FC14 & 0.3096 & 0.3455 & 0.333 & 0.6739 \\
FC15 & 0.3832 & $\mathbf{0 . 4 5 0 5}$ & 0.2962 & 0.5624 \\
FC16 & $\mathbf{0 . 7 7 3 4}$ & 0.0823 & 0.1949 & 0.3571 \\
FC17 & $\mathbf{0 . 8 6 5 5}$ & 0.0439 & 0.1492 & 0.2268 \\
FC18 & 0.0192 & 0.1632 & $\mathbf{0 . 7 1 5 5}$ & 0.4610 \\
FC19 & $\mathbf{0 . 4 7 4}$ & 0.0091 & 0.377 & 0.6331 \\
FC20 & 0.306 & -0.1591 & $\mathbf{0 . 6 2 1 2}$ & 0.4952 \\
FC21 & 0.873 & 0.0024 & 0.0928 & 0.2292 \\
FC22 & $\mathbf{0 . 3 2 3 5}$ & 0.2538 & $\mathbf{0 . 4 1 7 4}$ & 0.6567 \\
\hline
\end{tabular}

Fuente: Elaboración propia.

\begin{tabular}{ll}
\multicolumn{2}{c}{$\begin{array}{c}\text { Cuadro } 3 \\
\text { Configuraciones de factores } \\
\text { estratégicos }\end{array}$} \\
\hline Código & \multicolumn{1}{c}{ Categorías } \\
\hline F01 & $\begin{array}{l}\text { Focalización del mercado } \\
\text { F02 }\end{array}$ \\
F03 & Innovad del producto \\
\hline
\end{tabular}

Fuente: Elaboración propia.

calidad del producto e innovación del producto, encontradas en la presente investigación, son parcialmente coincidentes con las encontradas por Calderón et al. (2009a, 2009b, 2011a y 2011b): innovación en producto y excelencia operativa (calidad de producto); de forma similar, la configuración focalización del mercado es parcialmente coincidente con el recurso competitivo búsqueda de reputación.

Para la identificación de dimensiones que evalúen la efectividad organizacional se realizó un análisis factorial oblicuo, el cual identifica la existencia de dos factores significativos (autovalores $\geq 1$ ). 
Además se realizó la rotación de los factores por medio del método Oblimin (ver Tabla 2).

Para comprobar la bondad y ajuste del modelo propuesto, igual que en el constructo anterior, se realizó un análisis de correlación de los residuos de los ítems y un análisis de la matriz de correlación anti-imagen, que permite establecer que la muestra es adecuada para el constructo, y por tanto las cargas factoriales son estables.

El primer factor está compuesto por 8 de los 12 ítems, se caracteriza por la efectividad en ventas, la tasa de crecimiento, la cuota del mercado, las ganancias operativas, las ganancias por ratio en ventas, el flujo de caja de operaciones, el retorno sobre la inversión y los programas de reducción de costos, al cual se denomina maximización de la rentabilidad.

El segundo factor está compuesto por 4 de los 12 ítems, y se caracteriza por el desarrollo de nuevos productos, el desarrollo de mercados, las actividades de $I+D$, y el desarrollo de personal, por tanto se denomina inserción de mercados.

Luego de ser identificadas las configuraciones estratégicas por medio de un análisis factorial confirmatorio -CFAse pretende conocer la causalidad de éstas sobre el desempeño de la empresa por medio de un modelo de ecuaciones estructurales en dos etapas -SEM- ${ }^{6}$ (Anderson y Gerbing, 1988; Shook et al., 2004), el cual permite establecer la inci-
Tabla 2

Matriz de componentes principales rotados de los ítems de efectividad organizacional

\begin{tabular}{cccc}
\hline Variable & Factor $\mathbf{1}$ & Factor $\mathbf{2}$ & $\begin{array}{c}\text { Comuna- } \\
\text { lidades }\end{array}$ \\
\hline EO01 & $\mathbf{0 . 7 7 5 7}$ & 0.0834 & 0.3914 \\
EO02 & $\mathbf{0 . 6 7 2 4}$ & 0.2368 & 0.4918 \\
EO03 & $\mathbf{0 . 7 1 3 0}$ & 0.3073 & 0.3972 \\
EO04 & $\mathbf{0 . 7 5 5 7}$ & 0.2695 & 0.3563 \\
EO05 & $\mathbf{0 . 7 8 1 3}$ & 0.1976 & 0.3505 \\
EO06 & $\mathbf{0 . 7 3 4 1}$ & 0.1370 & 0.4423 \\
EO07 & $\mathbf{0 . 7 3 1 3}$ & 0.3206 & 0.3624 \\
EO08 & 0.1855 & $\mathbf{0 . 7 9 1 6}$ & 0.3390 \\
EO09 & 0.2542 & $\mathbf{0 . 7 4 6 7}$ & 0.3778 \\
EO10 & 0.1326 & $\mathbf{0 . 8 6 6 8}$ & 0.2311 \\
EO11 & $\mathbf{0 . 4 5 9 0}$ & 0.4285 & 0.6056 \\
EO12 & 0.2900 & $\mathbf{0 . 6 4 4 7}$ & 0.5003 \\
\hline
\end{tabular}

Fuente: Elaboración propia.

dencia de la estrategia empresarial sobre el desempeño organizacional. El modelo causal establecido es el siguiente:

$$
\eta_{i}=\beta \xi_{i}+\zeta_{i}
$$

En donde $\eta_{i}$ es el desempeño organizativo medido en variables latentes y observables, $\xi_{i}$ el factor estratégico según la tipología elaborada a partir de la escala de Dess y Davis (1984) y $\varsigma_{i}$ es la variable observada, asimismo el subíndice i representa las observaciones. El modelo en dos etapas inicialmente evalúa las propiedades psicométricas de las escalas utilizadas, como paso previo a la análisis. 
Efecto de la estrategia en el desempeño de la empresa...

Serna G., Héctor; Calderón H., Gregorio y Naranjo V., Julia

sustentación de las hipótesis. Es de anotar que el modelo está sujeto a las pruebas de bondad y ajuste, lo cual comprueba la insesgadez e inferencia de los resultados (Satorra y Bentler, 2001; Schermelleh et al., 2003).

A partir del modelo SEM en dos etapas se identifica que la estrategia desarrollada por la empresa a partir del peso otorgado a sus factores competitivos incide en el desempeño de la misma ( $p$ value $\leq 0.01$ ) exceptuando el indicador financiero ROE ( $p$ value>0.05). Específicamente se halla que la variable focalización del mercado incide significativamente sobre las ventas de la empresa ( $p$ value $\leq 0.05$ ), la variable calidad del producto incide significativamente sobre el indicador EBITDA ( $p$ value $\leq 0.05$ ), la variable innovación de producto incide sobre ROA ( $p$ value $\leq 0.05$ ), lo cual permite aceptar la hipótesis 2 (ver Tabla 3 ).

Las variables focalización del mercado y calidad del producto inciden sobre maximización de la rentabilidad ( $p$ value $\leq 0.001)$, y las variables focaliza- ción del mercado e innovación del producto inciden sobre inserción de mercados ( $p$ value $\leq 0.001$ ), resultados que validan la hipótesis 3 (ver Tabla 3 ).

La comprobación de los resultados en los modelos SEM están sujetos a la comprobación de la bondad y ajuste, ésta se evalúa por medio del nivel de significancia del modelo $^{7}$, es de anotar que para la valoración del modelo el nivel de significancia es una prueba necesaria pero no es suficiente para probar el ajuste del modelo-model fit-, por tanto es necesario evaluar la linealidad de los paráme$\operatorname{tros}^{8}$, la distribución de los términos del error $^{9}$ y la inexistencia de multicolineali$\operatorname{dad}^{10}$ y de heterocedasticidad ${ }^{11}$. Análisis que permite concluir que el modelo presenta bondad y sus resultados son ajustados.

La presente investigación sigue los comentarios de $\mathrm{Hu}$ y Bentler (1999) y McDonald y Ho (2002) para el uso de SEM, quienes establecen que el índice global del modelo puede ser sustituido por un detallado examen de discrepan-

7 Se realizó el Test $X^{2}(p$ value $<0,001)$ el cual demuestra la significancia de cinco de los seis modelos que evalúan la interacción de las configuraciones estratégicas con el desempeño organizacional desde lo cualitativo y lo cuantitativo.

8 Por medio del test de Wald se identifica que los parámetros son lineales y consistentes ( $p$ value $>0,05)$.

9 Se identifica por medio de un histograma de residuos y un análisis de distribución de probabilidad normal de los residuos la normalidad de los términos del error, asimismo se realizó el test de jarque-bera ( $p$ value $>0,05)$, con el cual se comprueba la inexistencia de perturbaciones estocásticas.

10 Se realizó el análisis de correlaciones de pearson de las variables latentes presentes en el modelo a un nivel de significancia del $1 \%$ y del $5 \%$, identificándose que ningún coeficiente presenta valores que puedan ser considerados como una correlación elevada $(r>0,70)$, por tanto puede afirmarse que no existe colinealidad en las variables del modelo de ecuaciones estructurales.

11 Se identifican que las varianzas de los términos del error de los individuos presentan homosedasticidad ( $p$ value $>0,05)$. 


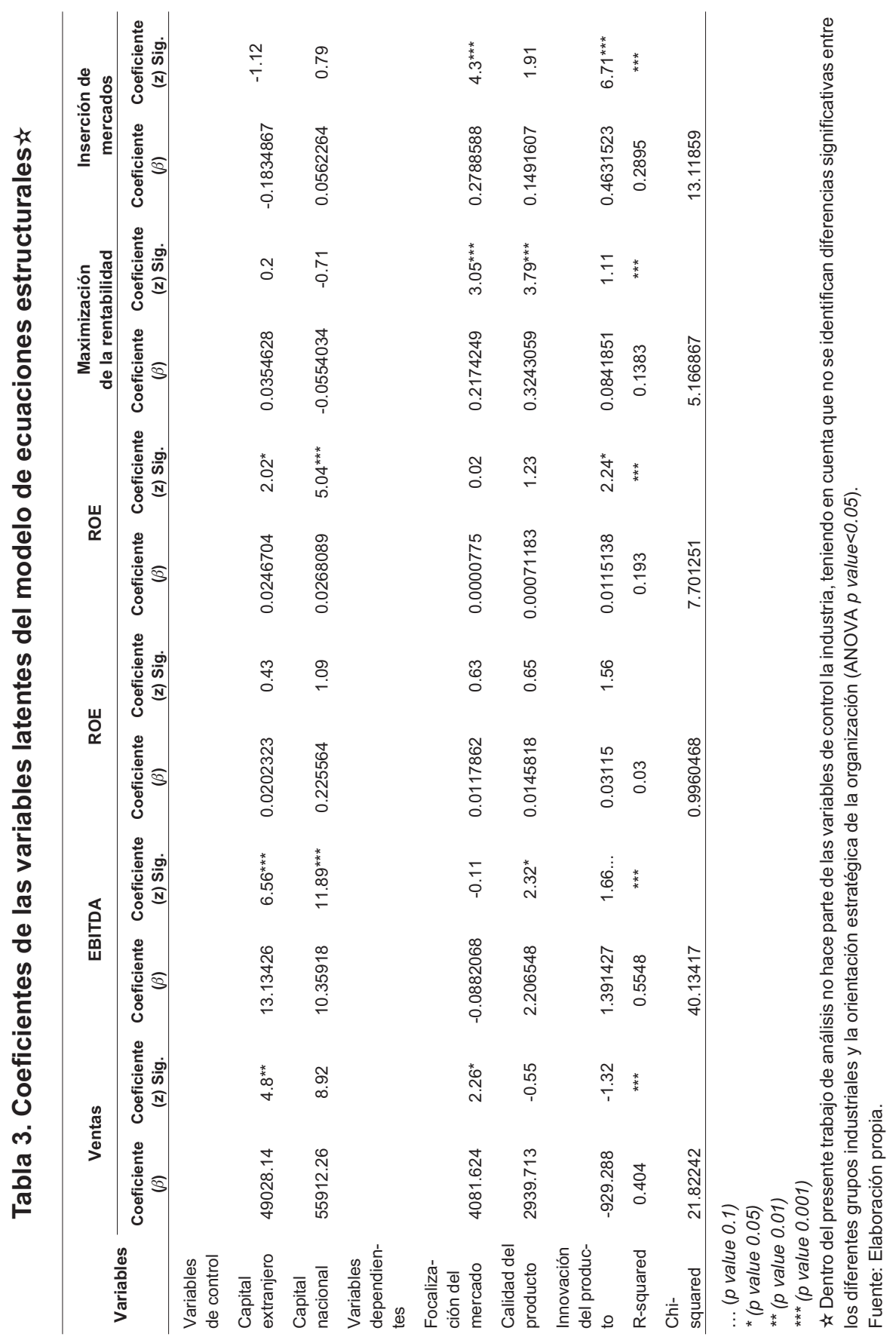


Efecto de la estrategia en el desempeño de la empresa...

Serna G., Héctor; Calderón H., Gregorio y Naranjo V., Julia

cias -aspecto que es valorado en la presente investigación, bajo el análisis de las matrices de correlación anti-imagen y las matrices de análisis de residuos generados por el CFA- lo cual permite una mayor aproximación a la realidad de la estimación (McDonald y Ho, 2002: 73).

Es importante reconocer que los resultados encontrados presentan aspectos convergentes en relación con investigaciones desarrolladas en otros contextos. Dess y Davis (1984) identifican, a nivel de intra-industria, la conformación de estrategias genéricas de acuerdo al peso dado a factores contingenciales, lo cual fue validado por Robinson y Pearce (1988). Asimismo Dess y Davis (1984) identifican que existe una relación significativa entre las estrategias diferenciación y grupos foco con el crecimiento en ventas y ROA, pero no encuentran evidencia para relacionar la estrategia disminución de costos con el desempeño de la empresa. Por su parte, Robinson y Pearce (1988) identifican que las empresas con una orientación estratégica clara tienen mejores indicadores en rentabilidad, nivel de ventas y ROA, que empresas sin orientación estratégica.

Asimismo estudios como los de Porter (1980), Robinson y Pearce (1988) y Camisón et al. (2007) plantean que la adopción de estrategias centradas en la calidad del producto y en la disminución de costos permiten alcanzar mayor volumen en ventas y mayores cuotas de mercado, lo que coincide con lo hallado en la presente investigación, una incidencia positiva y altamente significativa ( $p$ value $<0,001)$ del factor estratégico calidad del producto en la maximización de la rentabilidad de la empresa. Porter (1980: 62-
64) establece que un alto volumen de ventas se da por la posesión de una cuota elevada del mercado, generando per se una rentabilidad promedio mayor que el de la industria, esto se evidencia en la presente investigación con la incidencia positiva significativa $(p$ value $<0,05)$ de la variable focalización del mercado en las variables cualitativas ventas y maximización de la rentabilidad.

Es de acotar que los autores Porter (1980) y Camisón et al. (2007) establecen que estrategias de diferenciación permiten alcanzar nuevos mercados y generan mayor rendimiento del ROA que empresas sin orientación (Robinson y Pearce, 1988) aspectos que son identificados en el contexto colombiano.

Los resultados de Calderón et al. (2009a, 2011a y 2011b) señalan que las empresas colombianas presentan mayores diferencias en cuanto a la importancia que dan a factores relacionados con el marketing - mantenimiento de promoción y publicidad, canales de distribución, calidad de la publicidad, identidad de la marca e innovación en mercadeo-, lo que está en línea con los hallazgos de la presente investigación puesto que el factor estratégico focalización en el mercado es el que más incide sobre los indicadores de desempeño de la empresa.

Es importante resaltar que si bien existen trabajos que presentan relaciones causales entre estas variables, no utilizan medidas objetivas para valorar el desempeño, lo hacen a través de medidas subjetivas o de percepción (Camisón et al., 2007). El uso de medidas de percepción se valida en diferentes investigaciones (Dess y Davis, 1984; Venkatraman y Grant, 1986; Robinson y Pearce, 
1988; Chattopadhayay et al., 1999; Lyon et al., 2000) en razón a que para algunos investigadores la percepción de los directivos es más crítica con el desempeño que algunos indicadores objetivos (Chattopadhyay et al., 1999; Lyon et al., 2000).

Los trabajos que sí utilizan medidas objetivas, estudian relaciones a doble vía entre las variables y no identifican la incidencia que genera la estrategia sobre el desempeño (Porter, 1980; Dess y Davis, 1984; Robinson y Pearce, 1988).

Es de suma importancia para la investigación establecer una relación causal de la estrategia con los indicadores financieros -EBITDA, ROE, ROA, Ventas- $y$ demostrar la incidencia de la estrategia tanto en medidas subjetivas como objetivas, lo cual no es validado en investigaciones anteriores y permite tanto a la comunidad académica como a la empresarial establecer una relación directa en la forma en que se usan los factores competitivos para el incremento en la rentabilidad de la empresa.

\section{Conclusiones}

Los resultados presentan evidencias para aceptar las tres hipótesis formuladas: La relevancia dada a los factores competitivos genera estrategias genéricas en las empresas colombianas y la estrategia desarrollada por la empresa a partir del peso otorgado a sus factores competitivos, genera un impacto positivo en los indicadores financieros -ventas, EBITDA, ROE, ROA-y en la percepción del desempeño.

Desde los aspectos teóricos se destaca la relevancia de la teoría de re- cursos y capacidades para analizar la estrategia adoptada por la empresa, pero se identifica una ausencia de trabajos desde esta perspectiva que valoren la orientación al mercado y la relación con la estrategia, aspectos que han sido estudiados por la perspectiva de la organización industrial bajo modelos teóricos, y no bajo modelos empíricos.

Es interesante resaltar cuatro aspectos comparativos con estudios anteriores desarrollados por autores referenciados en el presente estudio. El primero tiene que ver con la modificación del método de rotación de factores del análisis factorial para los factores competitivos, el cual se realiza en el presente trabajo bajo el método oblimin, $y$ no mediante una transformación ortogonal, lo cual garantiza que los resultados no sean incorrelacionados, y permite la validación posthoc del modelo de ecuaciones estructurales -SEM-.

El segundo aspecto tiene que ver con la correspondencia parcial entre los factores encontrados en esta investigación con los hallados por Calderón en diferentes periodos. Las configuraciones estratégicas calidad del producto e innovación del producto, de esta investigación, se corresponden con los recursos estratégicos innovación en producto y excelencia operativa de los últimos. Por su parte, la configuración estratégica focalización del mercado se corresponde con el recursos estratégico búsqueda de reputación.

El tercer aspecto tiene que ver con los resultados evidenciados en las investigaciones, mientras en las investigaciones anteriores se identificaban grupos estratégicos en la industria colombiana de acuer- 
Efecto de la estrategia en el desempeño de la empresa...

Serna G., Héctor; Calderón H., Gregorio y Naranjo V., Julia

do al peso otorgado a sus factores competitivos y se identificaban relaciones entre los factores competitivos y el desempeño organizacional, en el presente trabajo se identifican configuraciones estratégicas en la empresa industrial colombiana y cómo estas configuraciones impactan de forma positiva y significativa el desempeño financiero y organizacional.

Asimismo la comparación de los resultados establece una clara conclusión, mientras en los trabajos anteriores las configuraciones estratégicas se centran más en los procesos relacionados en calidad y costos, en esta investigación, a partir del método de rotación de factores utilizado, se resaltan aspectos relacionados con la innovación y la focalización del mercado, sin dejar de lado la calidad, lo cual permite especificar que los procesos de innovación, marketing y calidad generan un mayor impacto en el rendimiento organizacional.

El cuarto aspecto tiene que ver con el modelo estructural propuesto, el cual permite dar un paso adelante, identificando causalidad de las variables de estudio sobre los indicadores financieros de la empresa y sobre la medida perceptual de desempeño organizacional.

De otra parte, es importante resaltar que las configuraciones establecidas en el presente estudio validan aspectos teóricos y permiten a las organizaciones colombianas inferir aspectos estratégicos, como que los procesos de racionalización y reducción de costos han pasado a un segundo plano y se presenta una fuerte incidencia del desarrollo de procesos de marketing -se destacan branding y la segmentación georeferenciada- $y$ calidad del producto.
En síntesis, el desarrollo de la investigación tuvo como limitante la recopilación de información primaria, la cual sólo permite aspectos metodológicos de corte transversal y sólo permite inferir la incidencia de la estrategia en el desempeño -identificación de los signos de los parámetros que acompañan las variables-y no permite valorar el impacto de la estrategia sobre la organización, aspectos que pueden ser analizados con modelos de panel dinámicos -valoración de varias unidades de observación a través del tiempo-, siempre y cuando se tenga información de las unidades de observación a través del tiempo. Es de acotar también que aunque la muestra es representativa -199 empresas- y se desarrollaron test para validar los resultados a nivel interindustrial, se identifica como limitante del análisis, la cual no permite hacer mayores comparaciones entre los tipo de industria dentro del modelo presentado.

\section{Referencias bibliográficas}

Akan, Obasi, Allen, Richard, Helms, Marilyn y Samuels Spralls (2006). Critical tactics for implementing Porter's generic strategies. En: Journal of Business Strategy. Vol. 27, No. 1. UK, Emerald Group Publishing Ltd. (43-53).

Anderson, Carl R. y Paine, Frank T. (1975). Managerial perceptions and strategic behavior. En: Academy of management journal. No.18. US, Academy of management (811-823).

Anderson, James y David Gerbing (1988). Structural equation modeling in practice: a review and recommend two step approach. En: Psychological bulletin. No. 103. US, University of California (453-460). 
Andrews, Kenneth (1971). The concepts of corporate strategy. US. Dow JonesIrwin.

Ansoff, H. Igor (1965). Corporate strategy.US. McGraw Hill.

Ansoff, H. Igor y Brandenburg, Robert G. (1971). A language for organizational design. En: Management science. Vol.17, No.2. US, University of Pennsylvania (717-731).

Aragón, Antonio (2004). Gestión de la formación en la empresa. Formación y cultura empresarial en la empresa Española. ES. Editorial Cívitas.

Aragón, Antonio, Barba-Aragón, Isabel y Raquel Sanz-Valle (2003). Effects of training on business results. En: International Journal of Human Resources Management. No.14. UK, University of Cardiff (956-980).

Barnard, Chester I. (1938). The functions of the executive. US: Harvard University Press.

Barney, Jay B. (1986). Strategic factor markets: expectations, luck, and business strategy. En: Management science. Vol. 42, No. 10. US, University of Pennsylvania (1231-1241).

Barney, Jay B. (1991). Firm resources and sustained competitive advantage. En: Journal of Management. No.17. US, Southern Management Association (99-120).

Barney, Jay B. (1997). Gaining and sustaining competitive advantage. US: Addison-Wesley publishing Co. Inc.

Barney, Jay B. y Robert E. Hoskisson (1990). Strategic groups: Untested assertions and research proposoals. En: Managerial and decision economics. Vol. 11, No. 3. US, Emory University (187-198).

Benett, Nathan, Ketchen, David y Eylssa B. Schultz (1998). An examination of factors associated with the integration of human. En: Human Resource Management. Vol. 37, No. 1 UK, Warwick Business School (3-16).

Bird, Allan y Schon Beechler (1995). Links between business strategy and human resource management strategy in U.S.-based Japanese subsidiaries: an empirical investigation. En: Journal of international business studies. Vol. 26, No. 1. US, Rutgers University (23-46).

Calderón, Gregorio, Naranjo, Julia C. y Claudia M. Álvarez (2009a). Orientación estratégica y recursos competitivos: un estudio en grandes empresas industriales de Colombia. En: Cuadernos de administración. Vol. 22, No. 38. CO, Pontificia Universidad Javeriana (849-72).

Calderón, Gregorio, Naranjo, Julia C. y Claudia M. Álvarez (2009b). Estrategia empresarial y gestión humana en empresas colombianas. $\mathrm{CO}$ : Unibiblios.

Calderón, Gregorio, Naranjo, Julia C. y Claudia M. Álvarez (2011a). Estrategia competitiva y desempeño organizacional en empresas industriales colombianas. En: Innovar. Vol. 20, No. 38. CO, Universidad Nacional de Colombia (13-26).

Calderón, Gregorio, Naranjo, Julia C. y Claudia M. Álvarez (2011b). Gestión empresarial en Colombia: Un aporte desde la administración. CO: Unibiblios.

Camelo, Carmen, Martín, Fernando, Romero, Pedro M. y Ramón Valle (2000). La estrategia empresarial y la gestión de los recursos humanos: un estudio del caso español En: Estudios financieros. No. 213. ES. Centro de Estudios Financieros (149-186).

Camelo, Carmen, Martín Fernando, Romero Pedro M. y Ramón Valle (2004). Human resources management in Spain: is it possible to speak of a typical 
Efecto de la estrategia en el desempeño de la empresa...

Serna G., Héctor; Calderón H., Gregorio y Naranjo V., Julia

model? En: International Journal of Human Resource Management. Vol. 15, No. 6, UK, University of Cardiff (935-958).

Camisón, César y Montse Boronat (2004). Factores determinantes del desempeño organizativo: efecto industria, incertidumbre y competencias distintivas. En: Investigaciones europeas de dirección y economía de empresa. Vol. 10, No. 3. ES, Academia Europea de Dirección y Economía de la Empresa (127-143).

Camisón, César, Garrigós, Fernando. J. y Daniel Palacios (2007). Estrategias competitivas y desempeño empresarial: Estudio comparativo de los modelos de Robinson y Pearce y Miles y Snow en el sector hotelero español. En: Investigaciones europeas de dirección y economía de la empresa. Vol. 13, No. 3. ES, Academia Europea de Dirección y Economía de la Empresa (161-182).

Carpenter, Mason y Gerard Sanders (2002). Top management team compensation: the missing link between CEO pay and firm performance? En: Strategic management journal. Vol. 23, No. 4. US, John Willey y Sons Ltd. (367-375).

Caves, Richard E. y Porter, M. E. (1977). From entry barriers to mobility barriers: conjectural decisions and contrived deterrence to new competition. En: Quarterly journal of economics. Vol. 91, No. 2. UK, Oxford University Press (241-262).

Chandler, Adam D. (1962) Strategy and structure. US. MIT Press.

Chattopadhayay, Prithviraj, Glick, Bill, Miller, Chet y Huber, George (1999). Determinants of executive beliefs: Comparing functional conditioning and social influence. En: Strategic management journal. Vol. 20 US, John Willey y Sons Ltd. (763-789).
Cool, Karel, y Dan Schendel (1987). Strategic group formation and performance: The case of the U.S. pharmaceutical industry, 1963-1982. En: Management Science.Vol.33, No. 9. US, University of Pennsylvania (1102-1124).

Deshpande, Rohit, Farley, James y Fred Webster (1993). Corporate culture, customer orientation and innovativeness in Japanese firms: A quadrate analysis. En: Journal of Marketing. Vol. 57, No. 1. US, American Marketing Association (23-37).

Dess, Gregory y Peter S. Davis (1984). Porter's (1980) generic strategies as determinants of strategic group membership and organizational performance. En: Academy of Management Journal. Vol. 27, No. 3. US, Academy of Management (467-488).

García-Borbolla, Amalia, Herrera, Jesús, Larrán, Jorge M., Sánchez, Gonzalo y Alfonso Suárez (2009). Análisis empírico de la influencia de la propiedad familiar sobre la orientación estratégica de las pequeñas y medianas empresas. En: Investigaciones europeas de dirección y economía de la empresa. Vol. 15, No. 1. ES, Academia Europea de Dirección y Economía de la Empresa (45-59).

Grant, Robert M. (1991). The resource-based theory of competitive advantage: Implications for strategy formulation. En: California Management Review Vol.33, No.1. US, University of California Berkeley (114-135).

Gupta, Anil K. y Vijay Govindarajan (1984). Business unit strategy, managerial characteristics and business unit effectiveness at strategy implementation. En: Academy of Management Journal. Vol. 27, No. 1. US, Academy of Management (25-41).

Hambrick, Donald C. y Mason, Phyllis A. (1984). Upper echelons: The organi- 
zation as a reflection of its top managers. En: The academy of management review. Vol. 9, No. 2. US, Academy of Management (193-206).

Hansen, Gary S. y Birger Wernerfelt (1989). Determinants of firm performance: the relative importance of economic and organizational factors. En: Strategic management journal. Vol. 10, No. 5. US, John Wiley y Sons Ltd. (399-411).

Hatten, Kenneth J. y Schendel, Dan E. (1977). Heterogeneity within an industry. En: Journal of Industrial Economics. Vol. 26, No. 2. US, Wiley Blackwell (97-113).

Hatten, Kenneth J., Schendel, Dan E. y Arnold Cooper (1978). A strategic model of the U.S. brewing industry: 1925-1971. En: Academy of Management Journal. Vol. 21. US, Academy of Management (592-619).

Hitt, Michael A., Ireland, Dave y Robert E. Hoskisson (2008). Administración estratégica, Competitividad y globalización, conceptos y casos. MX. Thompson.

Hill, Charles W. y Hill Jones (2005). Administración estratégica: un enfoque integrado. MX. McGraw Hill.

Hu, Li-tze y Peter Bentler (1999). Cutoff criteria for fit indices in covariance structure analysis: conventional criteria versus new alternatives. En: Structural equation modeling. Vol.6. US, University of California (1-55).

Hunt, Michael S. (1972). Competition in the major home appliance industry, 1960-1970. Disertación Ph.D. no publicada. US. Harvard University.

Ketchen, David J., Gombs, James G., Russell, Craig J., Shook, Chris, Dean, Michelle A., Runge, Janet, Lohrke, Franz T., Naumann, Stefanie E., Haptonstahl, Dawn E., Baker, Robert, Beckstein, Brenden A., Handler, Charles, Honig, Heather, y Stephen Lamoureux
(1997). Organizational configurations and performance: A meta-analysis. En: The academy of management journal. Vol. 40, No. 1. US, Academy of Management (223-240).

Lyon, Douglas W., Lumpkin, G. Thomas, y Gregory G. Dess (2000). Enhancing entrepreneurial orientation research: Operationalizing and measuring a key strategic decision making process. En: Journal of management. Vol. 26. US, Southern Management Association (1055-1085).

MacMillan, Ian C., Hambrick, Donald C. y Diana L. Day (1982) The product portafolio and profitability -APIMS- based analysis of industrial product businesses. En: Academy of management journal. Vol.25. US, Academy of Management (733-755).

McDonald, Roderick P. y Moon-Ho R. Ho (2002). Principles and practice in reporting structural equation analyses. En: Psychological methods. Vol. 7. US, American Psychological Association (64-82).

Miles, Raymond E., Snow, Charles C., Meyer, Alan D. y Herny J. Coleman Jr. (1978). Organizational strategy, structure and process. En: Academy of management review.Vol.3. US, Academy of Management (547-562).

Newman, Howard H. (1978). Strategic groups and the structure-performance relationship. En: Review of Economics and Statistics. Vol.60. US, Harvard Kennedy School (417-427).

Nunnally, Jum C. (1978). Psychometric theory.US. McGraw Hill.

Penrose, Edith T. (1959). The theory of the growth of the firm. US. Wiley.

Peteraf, Margaret A. (1993). The cornerstone of competitive advantage: a resource-based view. En: Strategic management journal. Vol.14. US, John Willey y Sons (179-191). 
Efecto de la estrategia en el desempeño de la empresa...

Serna G., Héctor; Calderón H., Gregorio y Naranjo V., Julia

Porter, Michael E. (1980). Competitive strategy: techniques for analyzing industries and competitors. US. Free Press.

Porter, Michael E. (1996). What is strategy? En: Harvard Business Review. Vol. 74, No. 6. US, Harvard University (61-78).

Quinn, Robert E. (1988). Beyond rational management: Managing the paradoxes and competing demandes of high performance. US. Jossey Bass.

Quinn, Robert E. y John Rohrbaugh (1981). A competing values approach to organizational effectiveness. En: Public Productivity Review. Vol. 5. US, M. E. Sharpe Inc. (122-140).

Quinn, Robert E. y John Rohrbaugh (1983). A spatial model of effectiveness criteria: Towards a competing values approach to organizational analysis. En: Management Science. Vol.29. US, University of Pennsylvania (363-377).

Robinson, Richard B. y John A. Pearce (1988). Planned patterns of strategic behavior and their relationship to business unit performance. En: Strategic management journal. Vol. 9. US, John Willey y Sons (43-60).

Rumelt, Richard P. (1991). How much does industry matter? En: Strategic management journal. Vol. 12. US, John Willey y Sons (167-185).

Sánchez, Gregorio y Antonio Aragón (2003). Top managers compensation, strategic orientations and firm performance: empirical evidence from Spanish firms. En: Management Research. Vol. 1. US, Iberoamerican Academy of Management (27-44).

Satorra, Albert y Peter M. Bentler (2001). A scaled difference chi-square test statistic for moment structure analysis. En: Psychometrika. Vol. 66. US, Psychometric Society (507-514).
Schendel, Daniel y Hofer Charles (1979). Strategic management: A new of business policy and planning. Ed. Little Brown: Boston.

Schermelleh-Engel, K.; Moosbrugger, H. y Müller, H. (2003). Evaluating the fit of structural equation models: test of significance and descriptive goodnessof-fit measures. En: Methods of psychological research online. Vol. 8, No. 2. GER, German Psychology Society (23-74).

Segal, Morley (1974). Organization and environment: A typology of adaptability and structure. En: Public administration review. Vol.35. US, American Society for Public Administration (212-220).

Selznick, Philip (1957). Leadership in administration: A sociological interpretation. US. Harper y Row.

Sheppeck, Michael A. y Jack Militello (2000). Strategic HR configurations and organizational performance. En: Human resource management. Vol. 39, No. 1. US, John Willey y Sons (5-16).

Shook, Christopher L., Ketchen, David J., Hult, G. Tomas M. y Michele Kacmar (2004). An assessment of the use of structural equation modeling in strategic management research. En: Strategic management journal. Vol. 25. No. 1. US, John Willey y Sons (397-404).

Venkatraman, Narayanan y Camillus, Jhon (1984). Exploring the concept of fit in strategic management. En: Academy of management review. Vol. 3. US, Academy of Management (613-624).

Venkatraman, Narayanan y Grant, Jhon (1986). Construct measurement in organizational strategy research: A critique and proposal. En: The academy of management review. Vol. 11, No. 1. US, Academy of Management (71-87) 
Wernerfelt, Birger (1984). A resource-based view of the firm. En: Strategic Management Journal. Vol. 5. US, Academy of Management (171-180).
Woo, Carolyny Y. y Arnold C. Cooper (1981). Strategies of effective low share business. En: Strategic management journal. Vol. 2. US, John Willey y Sons (301-318). 